\title{
BMJ Open Development and external validation of a dynamic nomogram for delayed cerebral ischaemia after aneurysmal subarachnoid hemorrhage: a study protocol for a multicentre retrospective cohort study
}

Ping Hu, ${ }^{1}$ Yuntao Li, ${ }^{1,2}$ Hongbo Zhang, ${ }^{3}$ Zhongzhou Su, ${ }^{2}$ Shancai Xu, ${ }^{4}$ Xuesong $\mathrm{Li},{ }^{5}$ Xu Gao, ${ }^{6}$ Yangfan Liu, ${ }^{7}$ Gang Deng, ${ }^{1}$ Yang Xu, ${ }^{1}$ Liguo Ye, ${ }^{1}$ Qianxue Chen (D) ${ }^{1}$

To cite: Hu P, Li Y, Zhang H, et al. Development and external validation of a dynamic nomogram for delayed cerebral ischaemia after aneurysmal subarachnoid hemorrhage: a study protocol for a multicentre retrospective cohort study. BMJ Open 2021;11:e051956. doi:10.1136/ bmjopen-2021-051956

- Prepublication history for this paper is available online. To view these files, please visit the journal online (http://dx.doi. org/10.1136/bmjopen-2021 051956).

$\mathrm{PH}$ and $\mathrm{YL}$ contributed equally.

$\mathrm{PH}$ and $\mathrm{YL}$ are joint first authors.

Received 01 April 2021

Accepted 30 November 2021

Check for updates

(C) Author(s) (or their employer(s)) 2021. Re-use permitted under CC BY-NC. No commercial re-use. See rights and permissions. Published by BMJ.

For numbered affiliations see end of article.

Correspondence to

Professor Qianxue Chen;

chenqx666@whu.edu.cn

\section{ABSTRACT}

Introduction Delayed cerebral ischaemia (DCI) caused by aneurysmal subarachnoid haemorrhage (aSAH) is the most frequent complication and typically contributes to poor neurological outcome or deterioration of patients' condition. Therefore, early accurate and effective prediction of $\mathrm{DCl}$ is urgently needed. This study aims to construct a dynamic nomogram for precisely calculating the risk of $\mathrm{DCl}$ in patients with aSAH. Internal validation of this tool is conducted using the training cohort, and independent external validation is completed by using other medical centre datasets.

Methods and analysis This study is a multicentre, retrospective, observational cohort study using data from patients with aSAH. The participants include all adult patients who received surgical treatment in neurosurgery of multiple medical centres from 1 September 2019 to 1 April 2021, including Renmin Hospital of Wuhan University, Huzhou Central Hospital, First Affiliated Hospital of Harbin Medical University, General Hospital of Northern Theatre Command and Affiliated Hospital of Panzhihua University. Clinical information is collected via the electronic medical record system, including demographic data, clinical state on admission and serum laboratory tests. Modified Fisher grade at admission, admission subarachnoid clot and cerebral oedema density, and residual postoperative subarachnoid clot density are determined using the electronic imagine record software. The primary outcome is $\mathrm{DCl}$.

Ethics and dissemination This study protocol was reviewed and approved by the Medical Ethics Committee of Renmin Hospital of Wuhan University, which is the principal affiliation of this study (approval number: WDRM2021-K022). The other Ethics Committees, including Huzhou Central Hospital (approval number: 20210800501), First Affiliated Hospital of Harbin Medical University (approval number: H202156), General Hospital of Northern Theater Command (approval number: Y2021060) and Affiliated Hospital of Panzhihua University (approval number: 202105002), also approved the protocol. The results of this research will be published in a peerreviewed medical journal.
Strengths and limitation of this study

- This study is the first multicentre clinical study to predict delayed cerebral ischaemia (DCI) after aneurysmal subarachnoid haemorrhage.

- This study is strictly designed, including rigorous inclusion/exclusion criteria, a single type of CT scanner, central blinded review, adequate sample size, missing value imputation and prospective trial registration.

- This study uses ShinyApps and R software to create a web-based dynamic nomogram to precisely calculate the risk probability of $\mathrm{DCl}$.

- One limitation of this study is that these variables in the training cohort may exist multicollinearity; least absolute shrinkage and selection operator method is, therefore, performed in the analysis, which will solve the high-dimensional data.

- Based on the limitation of retrospective datasets, a prospective study will be conducted in the future for further validation.

Trial registration number ChiCTR2100044448.

\section{INTRODUCTION}

Aneurysmal subarachnoid haemorrhage $(\mathrm{aSAH})$ is a severe condition that frequently suddenly occurs in some apparently healthy individuals. ${ }^{1}$ Surgical treatment options include microscopic clipping and coiling embolisation, which are effective intervention methods for patients with a ruptured aneurysm. $^{2}$ Delayed cerebral ischaemia (DCI) caused by $\mathrm{SAH}$ is the most prevalent complication, accounting for approximately $30 \%$ of all patients, ${ }^{3}$ which is a primary cause of mortality and disability and typically contributes to poor neurology outcome or 
deterioration of patients' condition. ${ }^{34}$ As a result, early accurate and effective prediction of DCI is urgently required.

The pathogenesis of DCI is complex. ${ }^{3}$ As previously stated, DCI potential mechanisms include large-vessel cerebral vasospasm, early brain injury, impaired autoregulation, microcirculatory dysfunction, micro thrombosis and cortical spreading depolarisation. ${ }^{5}$ Prophylactic measures of DCI primarily rely on blood pressure control, neurointensive care treatment and nimodipine administration. ${ }^{6}$ Early DCI detecting and intervention are highly critical; however, it is difficult to identify patients at high risk of developing DCI due to the ambiguity of potential predictors. At present, DCI diagnosis still relies on neurological manifestations and CT images. ${ }^{78}$ Previous studies have attempted to predict DCI according to patients' clinical information such as age, aneurysmal location, World Federation of Neurosurgical Societies (WFNS) classification, modified Fisher Scale and serum biochemical or cellular markers, including leucocytosis, platelet and $\mathrm{C}$ reactive protein. ${ }^{9-12}$ However, most of these predictors lack further verification or specificity. In a larger retrospective cohort study of 887 patients with $\mathrm{aSAH},{ }^{7}$ researchers finally modified age $>65$ years, Hunt and Hess (HH) grade of 4-5, modified Fisher Scale of 3-4, anterior circulation ruptured aneurysm, serum homocysteine level $>10 \mu \mathrm{mol} / \mathrm{L}$ and high blood pressure at admission. When the six factors were incorporated to construct a nomogram, a concordance index of 0.73 and 0.65 in the model training and validation cohorts, respectively, indicating the model's discrimination ability. ${ }^{7}$ Unfortunately, the nomogram lacks independent external validation.

Early accurate and effective DCI prediction can assist clinicians in administering appropriate drug therapy and selecting the best surgical procedures for patients with aSAH. ${ }^{2}$ Although a nomogram has been developed for predicting DCI and underwent internal validation, ${ }^{7}$ its effectiveness remains unclear in an independent external dataset. Moreover, the form of ordinary nomograms limited their utility in clinical practice. Among all clinical predictive models, a web-based dynamic nomogram is the most convenient and accurate tool to precisely calculate the risk probability of disease and facilitate individual decision-making. ${ }^{13} 14$ However, after a thorough assessment of relevant literature, we discovered no literature reports on using an externally validated dynamic nomogram to preoperatively predict risk probability of DCI.

Therefore, this study aims to construct a dynamic nomogram for precisely calculating DCI risk in patients with aSAH. Internal validation is conducted using the model training cohort, and independent external verification is completed by using other medical centre datasets. This tool will assist clinicians in making better clinical decisions and timely interventions to prevent or reduce unfavourable prognoses of DCI patients during a preoperative or postoperative stage of aSAH.

\section{METHODS AND ANALYSIS}

The study is a multicentre, retrospective, observational cohort study using data of patients with aSAH in electronic health record (EHR). The participant consists of all adult patients treated in the Department of Neurosurgery of several hospitals, including Renmin Hospital of Wuhan University, Huzhou Central Hospital, First Affiliated Hospital of Harbin Medical University, General Hospital of Northern Theater Command and Affiliated Hospital of Panzhihua University. This study is commenced on 1 December 2020, and the data collection of this research is terminated on 1 June 2021.

\section{Study population}

The study populations were enrolled in the Department of Neurosurgery of several hospitals, including Renmin Hospital of Wuhan University, Huzhou Central Hospital and Affiliated Hospital of Panzhihua University, from 1 September 2019 to 1 April 2021, as a model training cohort. Patients from First Affiliated Hospital of Harbin Medical University and General Hospital of Northern Theater Command served as independent external validation cohorts. According to guidelines, aSAH is diagnosed by head CT, CT angiography (CTA) or digital subtraction angiography. ${ }^{15}$

The inclusion criteria are as follows: (1) patients with aSAH admitted within only 24 hours after onset; (2) spontaneous aneurysmal SAH; (3) blood laboratory test and CT scan conducted within 24 hours after admission; (4) complete data in an electronic medical record system and electronic imagine record software; (5) surgical treatment performed within 72 hours after onset and (6) DCI occurred in 4-30 days after aSAH.

The exclusion criteria are as follows: (1) non-aneurysmal or traumatic SAH; (2) complicated with intracerebral bleeding; (3) complicated with vascular malformation; (4) acute infection on admission; (5) postoperative state on admission; (6) bilateral mydriasis or other permanent brain injuries on admission; (7) taking anticoagulants or corticosteroids within 1 month of hospitalisation and (8) non-surgical treatment.

\section{Data sources}

\section{Electronic medical record system}

Clinical information was collected via the electronic medical record system, including demographic data (sex, age, hypertension, smoking and alcohol consumption), clinical state on admission and serum laboratory tests (glucose, D-dimer, white blood cells (WBCs), neutrophils, lymphocytes and monocytes). The information on aneurysms was also recorded, including their numbers, location, length and neck size. WFNS and HH classifications are employed to measure clinical and neurological status at admission. ${ }^{1617}$ All patients received either a microsurgical clipping or coil embolisation as soon as possible. Postoperative routine therapies included haemostasis, analgesics, anti-inflammatory drugs and nimodipine to prevent vasospasm. Additionally, a CT scan of the head is 
conducted immediately following operation to determine any intracranial rebleeding or cerebral infarction.

\section{Electronic imagine record software}

All CT scans are completed by GE scanner (64-section Optimal CT680) without contrast enhancement. The following CT scanning parameters are described: tube voltage, $120 \mathrm{kVp}$; tube current modulation, $300 \mathrm{~mA}$; detector configuration, $64 \times 0.625 \mathrm{~mm}$; rotation time, $0.5 \mathrm{~s}$; slice thickness, $5 \mathrm{~mm}$; and collimation $10 \mathrm{~mm}$.

\section{Radiological features}

In this study, radiological variables included subarachnoid clot thickness, absence or presence of intraventricular haemorrhage, subarachnoid clot and cerebral oedema density, and residual subarachnoid clot density on postoperative CT. At admission, the modified Fisher grade represents the first two radiological features. ${ }^{18}$ The clot and oedema density are estimated on a region of interest (ROI), which is manually drawn on a representative CT slice by neurosurgeon and radiologist, who are blinded to patients' clinical information. The density of blood clots in the subarachnoid area on ROI (a circle with a diameter of 3-8 $\mathrm{mm}$ ) is assessed using mean Hounsfield unit (HU) value. The following subarachnoid cisterns/ fissures are employed to measure mean $\mathrm{HU}$ value: lateral Sylvian fissures, anterior interhemispheric fissures, medial Sylvian fissures, suprasellar cistern, ambient cistern and quadrigeminal cistern. ${ }^{19-21}$ In addition, ROI (a circle with a diameter of $1 \mathrm{~cm}$ ) of cerebral oedema is bilaterally and symmetrically drawn on a representative CT slice. The preceding methods of selection are as follows: (1) ROI is drawn on the thalamus and basal ganglia when blood clots are below the insular cortex and (2) otherwise, ROI is drawn on the bilateral centrum semiovale. ${ }^{22}{ }^{23}$ Moreover, since a previous study reported that clot volume and clearance rate served as independent predictors for vasospasm, the residual postoperative subarachnoid clot may have a strong prediction power on DCI. ${ }^{24} \mathrm{~A}$ CT scan is performed to measure the residual postoperative clot density according to the above-mentioned methods.

\section{Outcome assessment}

A blinded adjudication process for DCI is as follows.

Without knowledge of CT scans, one clinician confirms this in a retrospective review of each subject's clinical course. CT scans performed between 4 days and 30 days after SAH are retrospectively examined by two other neurosurgeons blinded to patients' clinical data. If the two clinicians discriminate in disagreement, they discuss and eventually reach the same conclusion.

At least one of the following statements defines DCI ${ }^{82}$ : 1. No other causes result in permanent or temporary focal neurological impairment between 4 days and 14 days following SAH (aphasia, apraxia, haemianopia or neglect).

2. At least two points decreased in the Glasgow Coma Scale (either on one of its components (eye open- ing, verbal response and motor response) or on total score).

3. A new low-density region on a representative slice associated with cerebral vasospasm between 4 days and 30 days after SAH is assumed as a recent infarction if it is not visible on admission and immediate postoperative CT scans. There are no other potential causes except cerebral vasospasm. In this study, cerebral vasospasm was determined using CTA and/or CT perfusion (CTP). ${ }^{26} 27$

\section{Statistical analysis}

Tables for baseline characteristics in both training and external validation cohorts

1. Patients demographic data: age (median), sex (male/ female), hypertension (yes/no), tobacco use (yes/no) and alcohol consumption (yes/no).

2. Clinical characteristics: WFNS grade (I-II/III/IV-V), $\mathrm{HH}$ grade (I-II/III/IV-V) and modified Fisher scale (MFS) (0-2/3-4).

3. Aneurysmal information: aneurysmal location (ACA/ $\mathrm{MCA} / \mathrm{ICA} / \mathrm{PCA} / \mathrm{ACoA} / \mathrm{PCoA} /$ others),

4. Aneurysmal number (single/multiple), aneurysmal mean size $(\mathrm{mm})$ (neck/length) and aneurysmal treatment (clipping/coiling).

5. Blood laboratory tests: glucose $(\mathrm{mmol} / \mathrm{L})$ counts, Ddimer $(\mathrm{mg} / \mathrm{L})$, WBC $\left(10^{9} / \mathrm{L}\right)$, neutrophil $\left(10^{9} / \mathrm{L}\right)$, lymphocyte $\left(10^{9} / \mathrm{L}\right)$, neutrophil-to-lymphocyte ratio $\left(10^{9} / \mathrm{L}\right)$ and monocytes $\left(10^{9} / \mathrm{L}\right)$ counts.

6. Admission CT value (HU): subarachnoid clot density (median) and cerebral oedema density (median).

7. Residual CT value (HU): subarachnoid clot density (median).

8. Complication after aSAH: hydrocephalus and DCI.

Kolmogorov-Smirnov test is performed to check that the variables are normally distributed. Continuous variables analysed using independent t-test or MannWhiney $\mathrm{U}$ test are presented as mean $\pm \mathrm{SD}$ or median with IQR. In contrast, categorical variables analysed by $\chi^{2}$ test or Fisher exact test are expressed as numbers (percentages).

\section{Variables selection}

Co-related variables are obtained by correlated heatmap or Spearman tests. When variables exhibit multicollinearity, the least absolute shrinkage and selection operator (LASSO) analysis is performed to select optimal factors. ${ }^{28}$ Otherwise, the conventional logistic regression method is deployed to select variables. According to grouping by DCI, univariate analysis is used to identify variables with a $p$ value of $<0.05$ among two cohorts. These variables are incorporated into multivariate analysis. Backward stepwise logistic regression based on Akaike information criterion is then conducted to identify independent predictors. ${ }^{29}{ }^{30}$ LASSO and logistic regression analysis are conducted using R software (https://www.rproject.org/). 


\section{Nomogram model development and evaluation}

A nomogram model is constructed via multivariate logistic regression based on variables selected by LASSO or backward stepwise analysis. Furthermore, a web-based dynamic nomogram is generated to precisely calculate the risk values for DCI occurrence. We use training and external validation cohorts to evaluate the nomogram's accuracy. Internal validation is conducted in the model training cohort using 1000 bootstrap resamples, and independent external validation is achieved by employing other central datasets. The receiver operating characteristic curve and C-index are used to reflect the model discrimination capacity. The calibration curve (1000 bootstrap resamples) and Hosmer-Lemeshow's $p$ value are applied to represent the calibration performance.

\section{Sample size}

In the current literature, events per variable (EPV) criterion, particularly an EPV of 10 or 15 , is extensively applied as the lowest limit for logistic regression models predicting a binary outcome. ${ }^{31}{ }^{32}$ Nine variables were included in the multivariate logistic regression based on pilot data from the principal affiliation site, including glucose, D-dimer, WBC, neutrophil, neutrophil-tolymphocyte ratio (NLR), WFNS, HH, SAH mean HU value and aneurysmal number. As a result, this study has an effective sample size of at least 90 according to an EPV of 10. DCI occurs following SAH in around $30 \%$ of cases worldwide. Accordingly, the training cohort should have at least 300 patients.

\section{Data management}

Due to a small number of cases, the data are stored using Microsoft Excel 2019 and IBM SPSS V.26.0. To maximise statistical power and minimise deviation by excluding patients with missing data from analysis, we use multivariate multiple imputations, based on five replications and a chained equation approach method to impute these missing data. ${ }^{33}$ If the missing data belong to continuous variables, linear regression is applied to the imputation, while logistic regression is conducted to categorical variables. ${ }^{33}$ These imputation methods are used for only prediction variables.

\section{Patient and public involvement statement}

This is a retrospective observational cohort study that the data for this research are derived from the EHR. Patients and the public are not involved in the design, conduct, reporting or dissemination plans of this study.

\section{ETHICS AND DISSEMINATION \\ Ethics approval statement}

This study protocol was prospectively registered with the Chinese Clinical Trials Registry. In addition, the study protocol was approved by the Medical Ethics Committee of Renmin Hospital of Wuhan University (approval number: WDRM2021-K022), which is the principal affiliation site of this study. The other Ethics Committees, including Huzhou Central Hospital (approval number: 20210800501), First Affiliated Hospital of Harbin Medical University (approval number: H202156), General Hospital of Northern Theater Command (approval number: Y2021060) and Affiliated Hospital of Panzhihua University (approval number: 202105002), also approved the protocol. All Medical Ethics Committees waived patient consent because the data will be derived from the EHR. All procedures performed in this study involving human participants followed the ethical standards of the institutional and national research committee and with the 1964 Declaration of Helsinki and its later amendments or comparable ethical standards. ${ }^{35}$ The results of this research will be published in a peer-reviewed medical journal.

\section{Author affiliations}

${ }^{1}$ Department of Neurosurgery, Renmin Hospital of Wuhan University, Wuhan, China

${ }^{2}$ Department of Neurosurgery, Huzhou Central Hospital, Huzhou, China

${ }^{3}$ Department of Neurosurgery, The Second Affiliated Hospital of Nanchang University, Nanchang, China

${ }^{4}$ Department of Neurosurgery, First Affiliated Hospital of Harbin Medical University, Harbin, China

${ }^{5}$ Department of Neurosurgery, Huizhou Third People's Hospital, Huizhou, China ${ }^{6}$ Department of Neurosurgery, General Hospital of Northern Theatre Command, Shenyang, China

${ }^{7}$ Department of Neurosurgery, Affiliated Hospital of Panzhihua University, Panzhihua, China

Contributors Study design: $\mathrm{PH}, \mathrm{YL}$ and QC. Literature search and data collection: $\mathrm{PH}, \mathrm{YL}, \mathrm{HZ}, \mathrm{ZS}, \mathrm{SX}, \mathrm{XL}, \mathrm{XG}$ and YL. Data analysis and statistical analysis: PH, GD, $Y X$ and $L Y$. Manuscript preparation, editing and review: PH, YL, GD, YX and QC. All authors read and approved the final manuscript.

Funding This work was supported by the National Natural Science Foundation of China (grant number: 82001311).

Competing interests None declared.

Patient and public involvement Patients and/or the public were not involved in the design, or conduct, or reporting, or dissemination plans of this research.

Patient consent for publication Not applicable.

Provenance and peer review Not commissioned; externally peer reviewed.

Open access This is an open access article distributed in accordance with the Creative Commons Attribution Non Commercial (CC BY-NC 4.0) license, which permits others to distribute, remix, adapt, build upon this work non-commercially, and license their derivative works on different terms, provided the original work is properly cited, appropriate credit is given, any changes made indicated, and the use is non-commercial. See: http://creativecommons.org/licenses/by-nc/4.0/.

ORCID iD

Qianxue Chen http://orcid.org/0000-0002-9413-1030

\section{REFERENCES}

1 Macdonald RL, Schweizer TA. Spontaneous subarachnoid haemorrhage. Lancet 2017;389:655-66.

2 D'Souza S. Aneurysmal subarachnoid hemorrhage. J Neurosurg Anesthesiol 2015;27:222-40.

3 Macdonald RL. Delayed neurological deterioration after subarachnoid haemorrhage. Nat Rev Neurol 2014;10:44-58.

4 Nilsson OG, Brandt L, Ungerstedt U, et al. Bedside detection of brain ischemia using intracerebral microdialysis: subarachnoid hemorrhage and delayed ischemic deterioration. Neurosurgery 1999;45:1176-85.

5 Budohoski KP, Guilfoyle M, Helmy A, et al. The pathophysiology and treatment of delayed cerebral ischaemia following subarachnoid haemorrhage. J Neurol Neurosurg Psychiatry 2014;85:1343-53. 
6 Francoeur CL, Mayer SA. Management of delayed cerebral ischemia after subarachnoid hemorrhage. Crit Care 2016;20:277.

7 Liu H, Xu Q, Li A. Nomogram for predicting delayed cerebral ischemia after aneurysmal subarachnoid hemorrhage in the Chinese population. J Stroke Cerebrovasc Dis 2020;29:105005.

8 Vergouwen MDI, Vermeulen M, van Gijn J, et al. Definition of delayed cerebral ischemia after aneurysmal subarachnoid hemorrhage as an outcome event in clinical trials and observational studies: proposal of a multidisciplinary Research Group. Stroke 2010;41:2391-5.

9 Lee H, Perry JJ, English SW. Clinical prediction of delayed cerebra ischemia in aneurysmal subarachnoid hemorrhage. J Neurosurg 2018:1-8.

10 Kasius KM, Frijns CJM, Algra A, et al. Association of platelet and leukocyte counts with delayed cerebral ischemia in aneurysmal subarachnoid hemorrhage. Cerebrovasc Dis 2010;29:576-83.

11 Srinivasan A, Aggarwal A, Gaudihalli S, et al. Impact of early leukocytosis and elevated high-sensitivity C-reactive protein on delayed cerebral ischemia and neurologic outcome after subarachnoid hemorrhage. World Neurosurg 2016;90:91-5.

12 Kubo Y, Ogasawara K, Kakino S, et al. Serum inflammatory adhesion molecules and high-sensitivity C-reactive protein correlates with delayed ischemic neurologic deficits after subarachnoid hemorrhage. Surg Neurol 2008;69:592-6.

13 Sun W, Li G, Song Y, et al. A web based dynamic MANA nomogram for predicting the malignant cerebral edema in patients with large hemispheric infarction. BMC Neurol 2020;20:360.

14 Wu Y, Han C, Wang Z, et al. An Externally-Validated dynamic nomogram based on clinicopathological characteristics for evaluating the risk of lymph node metastasis in small-size non-small cell lung cancer. Front Oncol 2020;10:1322.

15 Connolly ES, Rabinstein AA, Carhuapoma JR, et al. Guidelines for the management of aneurysmal subarachnoid hemorrhage: a guideline for healthcare professionals from the American Heart Association/american stroke association. Stroke 2012;43:1711-37.

16 Sano H, Satoh A, Murayama Y, et al. Modified World Federation of neurosurgical societies subarachnoid hemorrhage grading system. World Neurosurg 2015;83:801-7.

17 Zhang Y, Zhu X, Hou K, et al. Clinical outcomes of surgical clipping for intracranial aneurysms in patients with a Hunt and Hess grade 4 or 5. Arq Neuropsiquiatr 2016;74:478-81.

18 Frontera JA, Claassen J, Schmidt JM, et al. Prediction of symptomatic vasospasm after subarachnoid hemorrhage: the modified Fisher scale. Neurosurgery 2006;59:21-7.

19 Ahn S-H, Savarraj JP, Pervez M, et al. The subarachnoid hemorrhage early brain edema score predicts delayed cerebral ischemia and clinical outcomes. Neurosurgery 2018;83:137-45.

20 Woo PYM, Tse TPK, Chan RSK, et al. Computed tomography interobserver agreement in the assessment of aneurysmal subarachnoid hemorrhage and predictors for clinical outcome. $J$ Neurointerv Surg 2017;9:1118-24.

21 Ishihara H, Oka F, Kawano R, et al. Hounsfield unit value of interpeduncular Cistern hematomas can predict symptomatic vasospasm. Stroke 2020;51:143-8.

22 Kanazawa T, Takahashi S, Minami Y, et al. Early prediction of clinical outcomes in patients with aneurysmal subarachnoid hemorrhage using computed tomography texture analysis. J Clin Neurosci 2020;71:144-9.

23 Claassen J, Carhuapoma JR, Kreiter KT, et al. Global cerebral edema after subarachnoid hemorrhage: frequency, predictors, and impact on outcome. Stroke 2002;33:1225-32.

24 Reilly C, Amidei C, Tolentino J, et al. Clot volume and clearance rate as independent predictors of vasospasm after aneurysmal subarachnoid hemorrhage. J Neurosurg 2004;101:255-61.

25 Vergouwen MDI, Vermeulen M, Coert BA, et al. Microthrombosis after aneurysmal subarachnoid hemorrhage: an additional explanation for delayed cerebral ischemia. J Cereb Blood Flow Metab 2008;28:1761-70.

26 Letourneau-Guillon L, Farzin B, Darsaut TE, et al. Reliability of CT angiography in cerebral vasospasm: a systematic review of the literature and an inter- and intraobserver study. AJNR Am J Neuroradiol 2020;41:612-8.

27 Hoeffner EG, Case I, Jain R, et al. Cerebral perfusion CT: technique and clinical applications. Radiology 2004;231:632-44.

28 Riley RD, Snell KIE, Martin GP, et al. Penalization and shrinkage methods produced unreliable clinical prediction models especially when sample size was small. J Clin Epidemiol 2021;132:88-96.

29 Bursac Z, Gauss CH, Williams DK, et al. Purposeful selection of variables in logistic regression. Source Code Biol Med 2008;3:17.

30 Dunkler D, Plischke M, Leffondré K, et al. Augmented backward elimination: a pragmatic and purposeful way to develop statistical models. PLoS One 2014;9:e113677.

31 Moons KGM, de Groot JAH, Bouwmeester W, et al. Critical appraisal and data extraction for systematic reviews of prediction modelling studies: the charms checklist. PLoS Med 2014;11:e1001744.

32 Pavlou M, Ambler G, Seaman S, et al. Review and evaluation of penalised regression methods for risk prediction in low-dimensional data with few events. Stat Med 2016;35:1159-77.

33 Cummings P. Missing data and multiple imputation. JAMA Pediatr 2013;167:656-61.

34 Pedersen AB, Mikkelsen EM, Cronin-Fenton D, et al. Missing data and multiple imputation in clinical epidemiological research. Clin Epidemiol 2017;9:157-66.

35 World Medical Association Declaration of Helsinki. Ethical principles for medical research involving human subjects. JAMA 2013;310:2191-4. 